\title{
Long telomeres are associated with clonality in wild populations of the fissiparous starfish Coscinasterias tenuispina
}

This article has been corrected since Advance Online Publication and a corrigendum is also printed in this issue

\author{
A Garcia-Cisneros ${ }^{1}$, R Pérez-Portela ${ }^{2}$, BC Almroth ${ }^{3}$, S Degerman ${ }^{4}$, C Palacín ${ }^{1}$ and H Nilsson Sköld ${ }^{5}$
}

Telomeres usually shorten during an organism's lifespan and have thus been used as an aging and health marker. When telomeres become sufficiently short, senescence is induced. The most common method of restoring telomere length is via telomerase reverse transcriptase activity, highly expressed during embryogenesis. However, although asexual reproduction from adult tissues has an important role in the life cycles of certain species, its effect on the aging and fitness of wild populations, as well as its implications for the long-term survival of populations with limited genetic variation, is largely unknown. Here we compare relative telomere length of 58 individuals from four populations of the asexually reproducing starfish Coscinasterias tenuispina. Additionally, 12 individuals were used to compare telomere lengths in regenerating and non-regenerating arms, in two different tissues (tube feet and pyloric cecum). The level of clonality was assessed by genotyping the populations based on 12 specific microsatellite loci and relative telomere length was measured via quantitative PCR. The results revealed significantly longer telomeres in Mediterranean populations than Atlantic ones as demonstrated by the Kruskal-Wallis test $(K=24.17$, significant value: $P$-value $<0.001$ ), with the former also characterized by higher levels of clonality derived from asexual reproduction. Telomeres were furthermore significantly longer in regenerating arms than in non-regenerating arms within individuals (pyloric cecum tissue: Mann-Whitney test, $V=299, P$-value $<10^{-6}$; and tube feet tissue Student's $t=2.28$, $P$-value $=0.029$ ). Our study suggests that one of the mechanisms responsible for the long-term somatic maintenance and persistence of clonal populations is telomere elongation.

Heredity (2015) 115, 437-443; doi:10.1038/hdy.2015.43; published online 20 May 2015

\section{INTRODUCTION}

Although aging is observed in most organisms, there is a large degree of variation in the rate at which it occurs, at both the species and individual level. Telomere length has frequently been used as an aging marker because telomere caps normally become shorter during an organism's lifetime, not only primarily during DNA replication but also in association with other factors such as stress (Von Zglinicki, 2002; Epel et al., 2004; Kotrschal et al., 2007). Critically short telomeres trigger a signal prompting the cell to permanently stop dividing, which leads to the induction of cellular senescence (Herbig et al., 2006). Furthermore, long telomeres have been found to correlate with good health and higher life expectancies in several species, thereby also serving as an indicator of somatic fitness, which represents the boundary of aging diseases (Bize et al., 2009; Horn et al., 2010; Barrett et al., 2013).

During fission or fragmentation in asexual organisms, two or more separate individuals are formed, resulting in clonal offspring with genotypes identical to the parent and to each other. In wild asexual populations and after recurrent fissions, it is difficult to determine the age of an individual by morphological means, not only for the potential clone itself but also the original parental half. In these cases, genetic analyses can reveal the level of clonality within a population as well as the extent of a clone, thus providing information regarding its age and longevity (Ally et al., 2010). Indeed, extremely large and longlived clonally propagating populations exist, such as some sea grass species, with clones that are estimated to be 1000 year (Reusch et al., 1999) or more (Arnaud-Haond et al., 2012). In the case of a terrestrial tree, the age of some clones have been estimated as old as 10000 year (Ally et al., 2010) and in cold waters, clonal individuals of the coral Lophelia pertusa are estimated to be 4500-6000 year (Dahl et al., 2012). These estimations obtained for several plants and animal groups may in some way indicate the existence of mechanism to largely delay, or even resist, aging in particular clones although the evolutionary significance of these long-term resistance has not been clarify yet.

In sexually reproducing species, telomere length is restored during embryogenesis by the reverse transcriptase telomerase (Schaetzlein et al., 2004). However, little is known about aging in asexual organisms that propagate via fission or budding; many questions

${ }^{1}$ Department of Animal Biology (Invertebrates), University of Barcelona, and Biodiversity Research Institute (IRBIO), Barcelona, Spain; ${ }^{2}$ Center for Advanced Studies of Blanes (CEAB-CSIC), Acesso a la Cala Sant Francesc 14, Blanes, Girona, Spain; ${ }^{3}$ Department of Biological and Environmental Sciences, University of Gothenburg, Gothenburg, Sweden; ${ }^{4}$ Department of Medical Biosciensces, Umeå University, Umeå, Sweden and ${ }^{5}$ Sven Lovén Centre for Marine Sciences-Kristineberg, University of Gothenburg, Fiskebäckskil, Sweden

Correspondence: A Garcia-Cisneros, Department of Animal Biology, Barcelona University, 643 Diagonal Avenue, Barcelona 08028, Spain.

E-mail: alexgarcia.cis@gmail.com

Received 30 September 2014; revised 23 March 2015; accepted 13 April 2015; published online 20 May 2015 

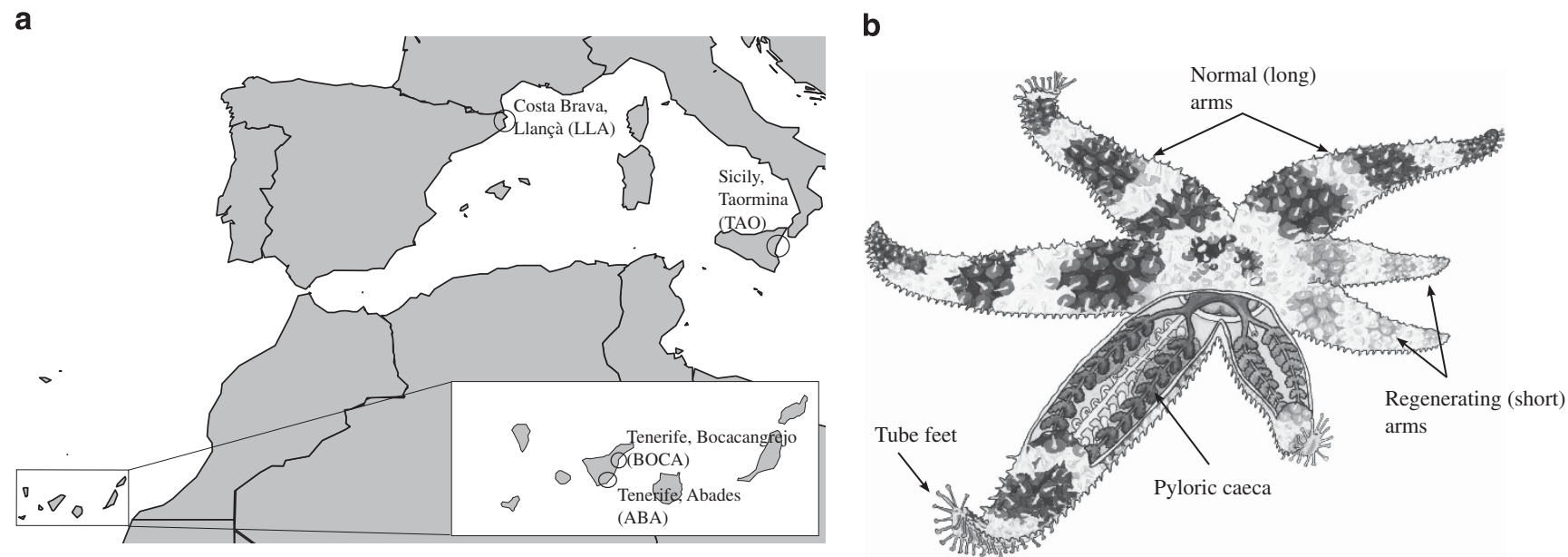

Figure 1 (a) Map of sampling locations, including those in Mediterranean Sea and Northeastern Atlantic Ocean. Circles highlight the three sampling areas. Two different populations were sampled in Tenerife. (b) Schematic anatomy of Coscinasterias tenuispina showing normal and regenerating arms.

remain unanswered as to whether they are able to fully maintain and/or restore their telomeres to persist over time or whether they undergo somatic aging (Sköld and Obst, 2011). In a study using laboratory cultures of two invertebrate species, upregulation of telomerase has been shown to at least partly restore telomeres in the clones of a flatworm (Tan et al., 2012). Telomerase is also upregulated during budding in the colonial ascidian Botryllus schlosseri (Laird and Weissman, 2004). On the other hand, experiments involving another colonial ascidian, Diplosoma listerianum, found that telomerase activity declined, telomeres shortened and the growth rate slowed after prolonged asexual duplication, indicative of long-term senescence in the studied clones (Sköld and Obst, 2011). There is, however, very limited information available regarding the effect of prolonged periods of asexual duplication on telomere length and aging in wild populations. Whether molecular aging occurs and how it could potentially be delayed in wild clones is still unknown.

Most asteroids have the ability to regenerate their body parts after autotomy or injuries, and about 26 species can reproduce asexually via fission (Emson and Wilkie, 1980). All four known species of the cosmopolitan genus Coscinasterias, commonly found in shallow waters, can reproduce both sexually and asexually via fission (Alves et al., 2002; Lawrence, 2013 among other references). When individuals of Coscinasterias reproduce sexually, they release planktotrophic larvae that remain in the water column for several weeks, with a high potential to colonize new habitats via dispersal (Karako et al., 2002). The species Coscinasterias tenuispina (Lamark 1816), widely distributed throughout the Atlantic Ocean and Mediterranean Sea, presents in some cases populations either consisting of individuals of only one gender (usually males) or with an unbalanced proportion of males and females (Alves et al., 2002; authors' unpublished data). The absence of one gender in some populations of this species, as well dominance of a few genotypes-according to allozyme analyses (Ventura et al., 2004) - suggests that maintenance of these populations takes place solely via asexual reproduction.

The aim of the present study was to assess the effect of asexual reproduction on relative telomere length in wild populations of the starfish C. tenuispina, and its implication for the long-term survival of populations with limited genetic variation. For this purpose, telomere length and its relationship with different levels of genetic diversity related to asexuality were assessed by using populations from two
Mediterranean and two Atlantic sites. Additionally, we explored the potential existence of mechanisms for telomere length control in somatic tissues by comparing regenerating and non-regenerating arms within a set of individuals.

\section{MATERIALS AND METHODS}

\section{Sampling}

Starfish of the species C. tenuispina (Supplementary Figure 1) were collected from four different European sites (Figure 1a), two in the Atlantic basin and two in the Mediterranean basin, with between 13 and 17 individuals collected per locality. The two Mediterranean sites, Llançà (Costa Brava, Northwestern Mediterranean) and Taormina (east of Sicily, Central Mediterranean), hereafter referred to as LLA and TAO, respectively, were sampled in autumn 2011. Both Atlantic sites, Bocacangrejo and Abades (BOCA and ABA, separated by $33 \mathrm{~km}$ ), were located near Tenerife (Canary Islands) and were sampled in the spring (June) of 2012 (Table 1). These four sampling locations were selected based on the abundance of the studied species and on the varying prevalence of individuals undergoing fission. The starfish were sampled at between 0 and $20 \mathrm{~m}$ depth by snorkeling or scuba-diving. Immediately after their removal from the sea, the animals were photographed on a millimeter-scaled table in order to determine body size. Tube feet, used for the starfish locomotion and substrate attachment, from the middle part of the longest arms of each individual were also collected (Figure 1b) and preserved in either RNAlater (Invitrogen, Life Technologies, Carlsbad, CA, USA; www.invitrogen.com) for telomere length analysis or absolute ethanol for microsatellite genotyping. The animals were then released back into the sea. All tissue samples were stored at $-20^{\circ} \mathrm{C}$ once in the laboratory prior to analysis. Body size was assessed as the longest diameter across the starfish using the ImageJ software program (Abramoff et al., 2004).

Additionally, both tube feet and pyloric caeca tissue (stomach extensions for processing and storage organ) were collected from the middle part of the longest (non-regenerating) and shortest (regenerating arm; when the length of this arm was $<50 \%$ of the longest arm) arms of 12 asymmetric specimens sampled at site LLA (Figure 1b, Supplementary Figure 1). Pyloric caeca tissue was considered in the present analysis because it is a distinct tissue that has further been shown to be involved in arm regeneration (Hernroth et al., 2010). Although gonads may be also considered for the telomere length analysis, they cannot be used in this study because more than $50 \%$ of the individuals of the species lack gonads, even during the reproductive season (Crozier, 1921).

\section{Genetic analyses using microsatellites}

Twelve microsatellite markers (m.ten1, m.ten6, m.ten13, m.ten14, m.ten19, m.ten24, m.ten25, m.ten27, m.ten30, m.ten31, m.ten32 and m.ten40) specifically designed for C. tenuispina (Garcia-Cisneros et al., 2013) were employed 
Table 1 Geographical coordinates and genetic information of the sampled Coscinasterias tenuispina populations

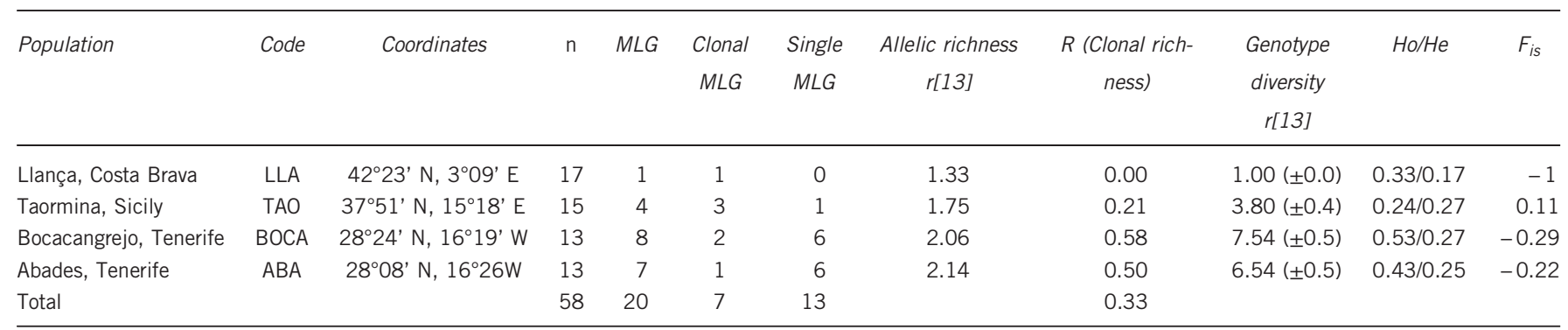

Abbreviation: MLG, multilocus genotypes.

Data presented include: sample size $(n)$, number of different MLGs, clonal MLGs (found in more than one individual) and single MLGs (found in only one individual), as well as allelic richness after rarefaction to 13 , clonal richness $(R)$, genotype diversity measured after rarefaction, observed and expected heterozigosity $(H o / H e)$ and inbreeding coefficient $\left(F_{\text {is }}\right)$.

for population genetic analyses (Table 1). Extractions and amplifications were performed using a REDExtract-N-Amp Tissue PCR Kit (Sigma-Aldrich, St Louis, MO, USA; www.sigmaaldrich.com). For PCR reactions we added $4 \mu \mathrm{l}$ of PCR Ready Mix, 4 pmol of each primer, between 10 and 50 ng of DNA, and ultrapure water for molecular use to a final reaction volume of $10 \mu \mathrm{l}$. Forward primers for each locus were labeled with a fluorescent dye as described in Garcia-Cisneros et al. (2013). Amplifications were performed on an S1000 Thermal Cycler Dual 48/48 Reaction Module (BioRad Laboratories, Hercules, CA, USA; www.bio-rad.com), with an initial step of 1 min denaturation at $96^{\circ} \mathrm{C}$ followed by 35 cycles of $96^{\circ} \mathrm{C}$ for $1 \mathrm{~min}, 49^{\circ} \mathrm{C}$ for $30 \mathrm{~s}$ and $72^{\circ} \mathrm{C}$ for $20 \mathrm{~s}$ and a final step at $72^{\circ} \mathrm{C}$ for $3 \mathrm{~min}$. Amplification products were analyzed on an ABI Prism 3730xl Genetic Analyzer (Applied Biosystems, Life Technologies, San Francisco, CA, USA; www.lifetechnologies.com) by the Scientific-Technical Services of the University of Barcelona. Allele size was estimated relative to an internal size standard 70-400 ROX (Bioventures Inc, Murfreesboro, TN, USA; www.bioventures.com) using the software program Peak-Scanner-96 (Applied Biosystems). The prevalence of identical genotypes, considered as potential clones, within and between populations was tested using GenoDive (Meirmans and Van Tienderen, 2004) and MLGsim (Stenberg et al., 2003). Both approaches were applied to count the number of individuals with identical multilocus genotypes (MLGs) and to calculate the likelihood of observing identical MLGs in a population due to sexual events $\left(P_{\text {sex }}\right)$. MLGsim calculations of the $P_{\text {sex, }}$, and their significance values, were based on 1000 random simulations. MLGsim set a critical value for MLGs because of sexual events at a minimum of 0.040 . Values obtained for MLGs of $C$. tenuispina were significantly lower than the critical value of 0.040 (see Table 1) pointing to clonal reproduction as the origin of identical MLGs. Genetic diversity was measured by calculating allelic richness, including all individuals from the populations even if they were considered the same clone, genotype diversity (synonym of clonal diversity) after rarefaction for each population, heterozygosity expected and observed and the inbreeding coefficient $\left(F_{\text {is }}\right)$ using the Hierfstat and Vegan packages (Dixon, 2003 Goudet, 2005) in the software program R v. 3.0.0.

In order to assess genetic differentiation between populations, we calculated values of the $D$ estimator (Jost, 2008), a statistic to measure differences in genetic structure between populations based on the allele frequencies, using the R package DEMEtics (Gerlach et al., 2010). The significance of these $D$ values was evaluated by performing 10000 permutations including all the individuals from the populations (Gerlach et al., 2010).

\section{Telomere measurements}

The relative telomere lengths in either tube feet or pyloric caeca tissue were measured for each specimen via a quantitative PCR (qPCR) method (Farzanehfar et al., 2008), and modified and optimized in our laboratory (Gothenburg, Sweden). Telomere length assessment via $\mathrm{QPCR}$ has already been validated (Grabowski et al., 2005; O'Callaghan et al., 2008). However, although qPCR telomere measurements are normalized based on single copy genes in humans and other model species, the lack of genomic data for non-model species such as C. tenuispina hinders the identification of nuclear genes without paralogs for normalization. For this reason, telomeric DNA measurements in the present study were performed relative to the total quantity of DNA in the samples.
Genomic DNA was extracted using the DNeasy Blood and Tissue kit (Qiagen, Venlo, Netherlands; www.qiagen.com) according to the manufacturer's instructions, and DNA concentrations assessed with a NanoDrop (Thermo Scientific, Waltham, MA, USA; www.thermoscientific.com) in triplicate to obtain an accurate value of DNA quantity. Absorbance ratios measuring DNA purity at 260/280 averaged approximately $1.9 \pm 0.1$, with the $260 / 230$ absorbance ratio also indicating acceptable sample purity $(1.8 \pm 0.2)$. Small differences in absorbance ratios were not correlated with final telomere length $\left(260 / 280: R^{2}=0.006, P=0.96 ; 260 / 230: R^{2}=0.001, P=0.99\right)$.

The amount of DNA to be added in the qPCR reaction was estimated from a standard curve obtained via a dilution series of a mixed sample DNA pool (10$0.0001 \mathrm{ng})$. The reaction efficiency calculated from the standard curve was $E=104.1 \%, R^{2}=0.997$. For the sample $\mathrm{qPCR}$ reaction, all DNA samples were adjusted to the same concentration, and $0.5 \mathrm{ng}$ of DNA was added for each PCR mix; this produced a concentration value falling well within the linear range of the mixed sample standard curve.

Telomere analyses examining a broad range of species have indicated that the TTAGGG sequence is conserved among deuterostomes (Gomes et al., 2010); the primers used for the qPCR reaction in C. tenuispina were therefore those described for humans in Farzaneh-far et al. (2008).

(forward) 5' CGGTTTGTTTGGGTTTGGGTTTGGGTTTGGGTTTGGGTT 3', and (reverse) 5' GGCTTGCCTTACCCTTACCCTTACCCTTACCCTTACCCT 3'.

Concentrations used for the forward and reverse primers were 100 and 200 nM, respectively. A 10- $\mu$ l KAPA SYBR FAST qPCR Kit (Kapa Biosystems, Wilmington, MA, USA; www.kapabiosystems.com) was employed as a Mastermix, with $3 \mu \mathrm{l}$ of water added for a final PCR volume of $20 \mu \mathrm{l}$ following the protocol described by Carney Almroth et al. (2012). Telomeres were amplified in triplicate for each sample to ensure accurate measurements using the following qPCR protocol cycle: $3 \mathrm{~min}$ at $95^{\circ} \mathrm{C}$ followed by 25 cycles of $15 \mathrm{~s}$ at $95^{\circ} \mathrm{C}$ and $1 \mathrm{~min}$ at $56^{\circ} \mathrm{C}$. The final step comprised 81 cycles of temperature increase from 55 to $95^{\circ} \mathrm{C}$ in order to generate a melt curve, indicating the presence of a single product. Relative telomere length is represented as a cycle threshold value (Ct value), which is inversely proportional to telomere amount; longer telomeres thus produce an earlier detectable signal than shorter telomeres. All measurements were analyzed in seven PCR plates, with the mixed sample DNA dilution series included on each plate as an internal standard; Ct values for these differed by less than $1.5 \%$ between plates and were adjusted for.

Telomere length verification was performed in an independent laboratory (Umeå, Sweden) using a similar protocol for telomere length qPCR (Cawthon, 2002). DNA from 11 samples were send to Umeå and DNA concentration were re-measured by the Nanodrop instrument (Thermo scientific). Each qPCR reaction contained: $17.5 \mathrm{ng}$ DNA (diluted in TE/E. coli buffer), 0.1- $\mu \mathrm{M}$ forward primer, 0.9- $\mu \mathrm{M}$ reverse primer, $1 \times$ PCR Buffer 2, $1.7 \mathrm{~mm}-\mathrm{MgCl} 2,2.5 \mathrm{~mm}-\mathrm{DTT}$, 0.2 mm-dNTP, 150-nм ROX, 0.2X SYBR and 0.625 U AmpliTaq Gold (Applied Biosystems).

Telomere primer sequence $\left(5^{\prime}-3^{\prime}\right)$ Forward: CGGTTTGTTTGGGTTTG GGTTTGGGTTTGGGTTTGGGTT, Reverse: GGCTTGCCTTACCCTTACCC TTACCCTTACCCTTACCCT. Each sample was analyzed two separate times in triplicates on the ABI7900HT instrument (Applied Biosystems). 
Statistical analyses

To compare body size between specimens from different populations, a nonparametric Mann-Whitney test was performed since the data did not match our prior expectation of homoscedascity (Bartlett test $=35.97$, and signification value, $P$-value $<10^{-7}$ ). As body size data also did not match normality (Shapiro-Wilk test; $W=0.93, P$-value $=0.0048$ ), a Spearman correlation was used to test if telomere lengths were correlated to body size. A mixed model analysis of variance, with telomere length (expressed as the Ct value) as a dependent variable, was performed using 'basin' (Atlantic and Mediterranean basins) as a fixed factor, and 'population' and 'MLGs' as random factors. A non-significant effect was found for the MLGs $(P$-value $>0.98)$ and was therefore not considered for further analyses (see Supplementary Figure 3). Since our data did not match normality (Shapiro-Wilk test; $W=0.96$, $P$-value $<0.01$ ), a Kruskal-Wallis test of the telomere length was performed between the different sites, and a Mann-Whitney test between basins. Spearman correlation was used to test the relationship between genetic diversity and mean telomere length.

Potential differences in telomere length between regenerating and nonregenerating arms within individuals were tested using a mixed model involving the logarithmic transformation of Ct values, 'tissue type' as a factor, and interaction with 'regeneration'. Moreover, we separately tested the effects of regeneration for both tissue types via a paired-data student's $t$-test for tube feet, and a paired Mann-Whitney test for pyloric caeca telomere length since the data did not match normality (non-normal distribution; Shapiro-Wilk test; $W=0.8482, P$-value $=0.002$ )

All statistical analyses of telomere length and box plots were performed in $\mathrm{R}$ v. 3.0.0.

\section{RESULTS}

Genetic diversity and genetic distance between localities

The low and significant values ( $P$-values) of the $P_{\text {sex }}$ (Table 2) indicated that the identical genotypes observed among individuals are a consequence of clonal propagation in this species. The studied populations of $C$. tenuispina presented different levels of allelic richness and clonality as defined by identical genotypes (Table 1). Populations from the Atlantic Ocean were genetically more diverse than those from the Mediterranean Sea, presenting 12 out of the 13 single multilocus genotypes (MLGs) found in the whole study area. Furthermore, excess of heterozygotes were found in all populations except Taormina. The $D$ estimator, used to assess differences in genetic structure between populations, revealed significant genetic differences among all four populations here analyzed (Table 3). Large and significant differences were recorded between the two Mediterranean populations, with the latter characterized by a higher prevalence of genetic clones compared with the Atlantic populations. Indeed, only one multilocus genotype was detected at Llançà (Table 1).

\section{Differences in telomere length and body size between and within populations}

Telomeres were significantly longer in the Mediterranean than in the Atlantic starfish populations as shown by the Kruskal-Wallis test ( $K=24.17$, significant value: $P$-value $<0.001$ ) (Figures $2 \mathrm{a}$ and $\mathrm{b}$ ). When the four populations were analyzed separately, significantly longer telomeres were again observed in the two Mediterranean populations (Kruskal-Wallis test; $K=37.03, P$-value $<0.001$ ), with individuals from Llançà presenting the longest telomeres (Figure 2b). Telomere length measurements were double-checked and verified by an independent laboratory (Umeå, Sweden), and the result showed a strong correlation between the measurements between both independent sets of analyses (value of the correlation for the regression: $R^{2}=0.88$ ), (Supplementary Figure 2).

Genotype diversity (also expressed as clonal diversity) in this species depends on the relative ratio of fission (asexual reproduction) versus
Table 2 Different MLGs found in more than one individual in the four localities

\begin{tabular}{lcrcc}
\hline Clonal MLG & Locality & $\mathrm{n}$ & $\mathrm{P}_{\text {sex }}$ & P-value \\
\hline MLG 1 & LLA & 17 & $<10^{-14}$ & $0.000^{*}$ \\
MLG 2 & TAO & 5 & 0.000 & $0.000^{*}$ \\
MLG 4 & TAO & 3 & $<10^{-8}$ & $0.007^{*}$ \\
MLG 8 & TAO & 6 & $<10^{-15}$ & $0.000^{*}$ \\
MLG 9 & BOCA & 2 & $<10^{-10}$ & $0.000^{*}$ \\
MLG 11 & BOCA & 5 & 0.000 & $0.000^{*}$ \\
MLG 12 & ABA & 7 & $<10^{-14}$ & $0.000^{*}$
\end{tabular}

Abbreviations: ABA, Abades; BOCA, Boca Cangrejo; LLA, Llançà; multilocus genotypes, MLG; TAO, Taormina.

Data presented include the number of individuals sharing the same MLG $(n)$, the probability of obtaining the same MLG from different sexual events $\left(P_{\text {sex }}\right)$ and the associated $P$-value of $P_{\text {sex }}$ *Significant when $P$-values $<0.01$.

Table 3 Values of the D genetic differentiation estimator between populations of Coscinasterias tenuispina

\begin{tabular}{llll}
\hline & TAO & LLA & BOCA \\
\hline LLA & $0.167^{*}$ & & \\
BOCA & $0.159^{*}$ & $0.233^{*}$ & \\
ABA & $0.143^{*}$ & $0.148^{*}$ & $0.138^{*}$
\end{tabular}

Abbreviations: ABA, Abades; BOCA, Boca Cangrejo; LLA, Llançà; TAO, Taormina. *Indicates significant $P$-values $<0.01$

sexual reproduction. Here, populations with lower genotype diversity, and therefore higher fission rates had longer telomeres at the population level, as demonstrated by a significant correlation (Pearson correlation: $R=0.99, P$-value $<0.007)$. In Figure 3 , it is presented the high correlation between mean of genotype diversity per population and the $\mathrm{Ct}$ value (which is inversely proportional to telomere length).

Populations analyzed showed differences in the mean body size of the starfish, demonstrated by a significant value of the Mann-Whitney test $\left(W=740, P\right.$-value $<10^{-7}$ ), with considerably larger specimens observed in the Atlantic populations (mean body size $=6.36 \pm$ s.d. $3.8 \mathrm{~cm}$ ) than in those from the Mediterranean Sea (mean body size $=2.81 \pm$ s.d. $1.1 \mathrm{~cm}$ ). However, telomere length did not depend on the starfish body size, as demonstrated by the absence of correlation between these two variables (correlation value: $\rho=0.065$, $P$-value $=0.63$, non-significant) (Supplementary Figure 3).

\section{Telomere length in relation to arm regeneration}

Our results showed that telomere length was significantly longer in regenerating (short) arms than in non-regenerating (long) arms (Figure 1b, Supplementary Figure 1), as demonstrate by the significance of the different tests applied $(F=52.26, P$-value $<0.001)$, for both tissue types analyzed, tube feet (Student's- $t=2.28, P$-value $=$ 0.029 ) and pyloric cecum tissue (Mann-Whitney, $V=299, P$-value $\left(10^{-6}\right.$ ) (Figure 4). Pyloric cecum telomeres were always longer in regenerating arms in all individuals, while 8 out of 12 specimens displayed longer telomere lengths in regenerating arm tube feet.

\section{DISCUSSION}

Life expectancy in clonal lineages remains unclear due the lack of understanding of different phenomena that would influence on its time survival. Firstly, we do not know the real consequences of the accumulation of somatic mutations and their deleterious effects on the 
a

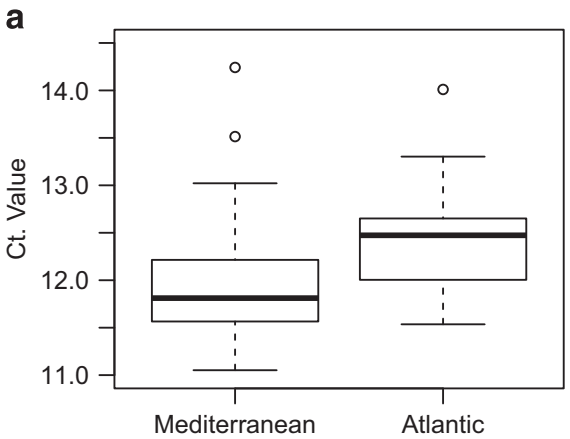

b

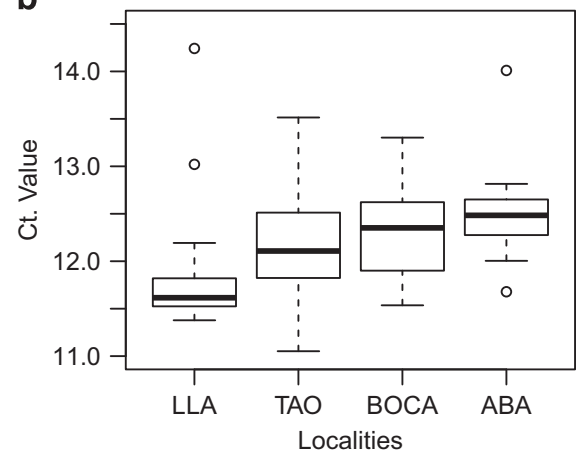

Figure 2 Box plots of telomere qPCR Ct values obtained from tube feet for the Coscinasterias tenuispina populations from different seas and localities: (a) Ct values of grouped Atlantic and Mediterranean populations, and (b) Ct values of each separate locality. Lower Ct values indicate longer telomeres. Boxes are represented by the first and third quartile, the dark line is the median, and dots are outliers. Llançà (LLA), Taormina (TAO), Abades (ABA) and Bocacangrejo (BOCA).

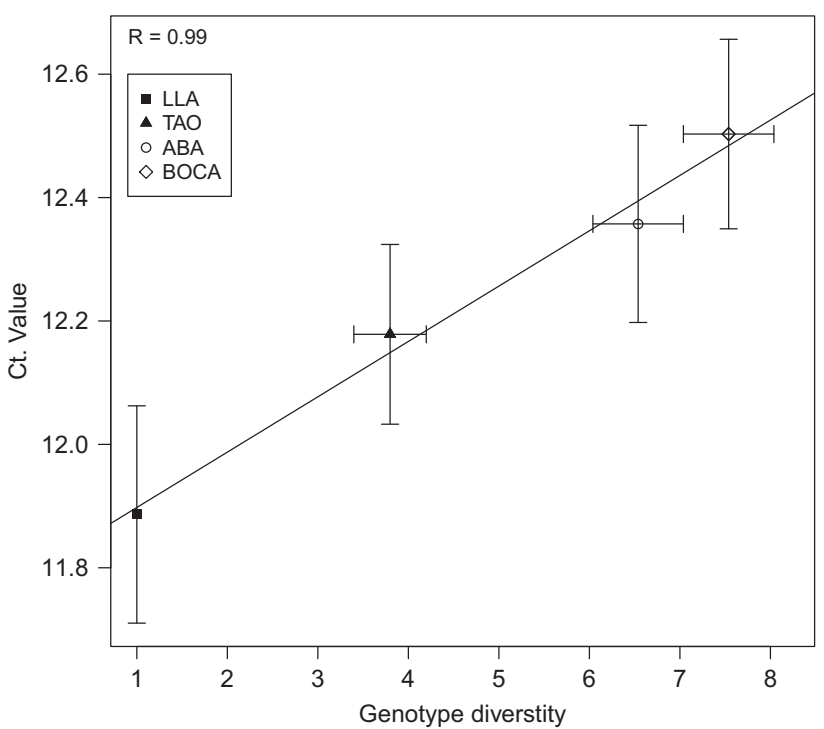

Figure 3 Correlation between relative telomere length in tube feet (telomere qPCR Ct Value) and genotype diversity for the four Coscinasterias tenuispina populations analyzed. Horizontal and vertical bars represent the standard error for genotype diversity and relative telomere length, respectively. The relative telomere length was represented as the mean value per locality.

individual and their clonal offspring. Secondly, we ignore the real effect in wild populations of the lack of genetic diversity for adaptive potential, and finally the mechanisms to avoid senescence. The first two difficulties are usually overcome when the species are able to maintain sex, even at low rates or sporadic events, by combining genomes and eliminating phenotypic expression of deleterious mutations when recessive. Here, in this study, we shed some light on the third problem, evidencing telomere elongation during asexual reproduction of the starfish C. tenuispina.

Our work represents the first study to explore the potential implications of asexual reproduction on relative telomere length in wild populations of a clonal starfish. To date, most ecological studies examining telomere length and/or telomerase activity have focused on obligate sexually reproducing species or clonal organisms maintained in laboratory cultures (see examples in Klapper et al., 1998; Horn et al., 2010; Ojimi and Hidaka, 2010; Sköld and Obst, 2011; Carney

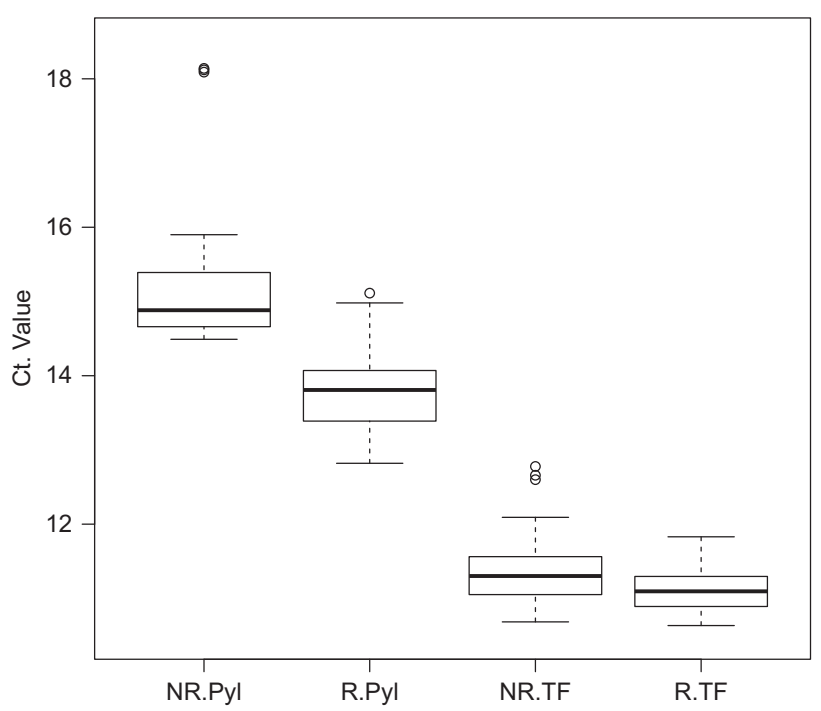

Figure 4 Box plot of Coscinasterias tenuispina telomere qPCR Ct values in regenerating and non-regenerating arms for two different tissues from individuals of Llança: pyloric cecum (Pyl) and tube feet (TF). Boxes are represented by the first and third quartile, the dark line is the median, and dots are outliers. Pyloric cecum from non-regenerating arms (Pyl NR); pyloric cecum from regenerating arms (Pyl R); tube feet from non-regenerating arms (TF NR); tube feet from regenerating arms (TF R).

Almroth et al., 2012; Tan et al., 2012), and no previous study based on telomeres and aging has been conducted on wild clonal populations of any species.

Longer telomeres were recorded in the Mediterranean populations of the starfish C. tenuispina, while these specimens also significantly smaller in size. Although shorter telomeres were observed in the Atlantic populations characterized by a larger mean body size, our results did not detect any correlation between the two variables. This finding is consistent with the lack of age-related telomere shortening demonstrated for other marine species, including sea urchins and lobsters, and may be attributed to high phenotypic plasticity in body size and/or to continuous telomerase activity throughout their lifespan (Klapper et al., 1998; Ebert et al., 2008). Nevertheless, telomere length is regarded as an indicator of health and somatic fitness, and its variation observed in our populations may be influenced by both 
inherited and/or environmental components (Epel et al., 2004). Thus, populations of $C$. tenuispina comprising only one clone may be healthily maintained, including those in Llançà, which were also characterized by the longest telomeres.

The longer telomeres found in regenerating compared with longer non-regenerating arms are indicative of telomere elongation and the preservation of chromosome ends in somatic tissue over the asexual cycle. These results may explain the positive correlation between telomere length and level of clonality. Asexual reproduction via fission has been proposed as more prevalent in small specimens of Coscinasterias (Emson and Wilkie, 1980), which is also consistent with our results and observations. It is therefore possible that the key to retain long telomeres in these starfish is to frequently undergo fission. On the other hand, fission may be influenced by the environment, either directly, for example, by different temperature regimes between the Mediterranean and Atlantic basins here analyzed, or indirectly, for example, by growth limitation and therefore it could be more prevalent in such situations (Haramoto et al., 2007). Therefore, in C. tenuispina, longer telomeres and greater somatic fitness may be triggered by unstable environmental conditions in wild populations.

Elongation of telomeres in populations of $C$. tenuispina may be one of the mechanisms related to the absence of senescence and genetic defects associated to prolonged periods of asexual propagation. Studies investigating terrestrial species have demonstrated that both telomere length and telomere erosion are predictors of survival and somatic fitness (Bize et al., 2009; Horn et al., 2010), with the combination of long telomeres and telomere elongation in regenerating tissues potentially providing these clonal starfish a high probability of survival. Although the results of a previous study examining a colonial ascidian suggest that passing through a sexual reproductive phase is required in order to avoid senescence after prolonged periods of asexual budding (Sköld et al., 2011), it is not known how generally this finding can be applied to other species. Despite the fact that asexual reproduction facilitates clonal dispersion and renewal, it depends on mitotic divisions, which may increase the accumulation of somatic mutations, the Muller ratchet phenomena. Different studies with asexual species already revealed accumulation of somatic mutations on nonsynonymus position in clonal lineages compared with their nonclonal sibling species (Paland and Lynch, 2006; Barraclough et al., 2007). Unfortunately, the negative effects of deleterious mutations in wild populations of clonal species have never been fully investigated or proved, and these effects have been only supposed, but asexual lineages persist over short evolutionary periods (Schwander and Crespi, 2009). However, older clones of aspen species has been found to exhibit a significant reduction in reproductive performance associated with male sexual fitness decline, suggesting that at least some long-lived clonal organisms may be vulnerable to senescence over long periods of time (Ally et al., 2008). A hypothesis to explain the excess of heterozygosity found in the studied populations of C. tenuispina may be a positive selection of heterozygotes to keep genetic diversity, besides having greater individual adaptability by high phenotype plasticity (Hörandl, 2009; Goudie et al., 2012). Nevertheless, our current results cannot actually test this hypothesis, and the heterozygotes excess found in all populations may be results from other stochastic processes that have not been controlled in this study. In other organisms as plants that maintain asexual lineages has been observed that polyploidy and hybridization commonly generate heterozygotes. In other species as fur seals, inbreeding is avoided by an active selection from females for non-relative males or heterozygotes (Hoffman et al., 2007). However, we do not have evidences of any of these processes, and positive selection of heterozygotes is the only hypothesis that can be presented here, but further studies on this particular point may shed light on it.

Although the molecular mechanisms responsible for telomere elongation and its preservation in C. tenuispina remain unknown, studies examining other asexual and sexual organisms indicate a pivotal role for telomerase in the telomere length regulation of somatic cell lineages. In Asterias rubens, a sexually reproducing starfish, telomerase activity is high throughout the animal irrespectively of mitotic activity and there was no difference in telomerase activity nor telomere length between regenerating and non-regenerating arms (Hernroth et al., 2010), but clonal starfishes may have higher telomerase activities after fission as other asexual species. In flatworms, maintenance of somatic telomere length seems to be an adaptation of asexual but not sexual strains, and is based on different levels of telomerase activity (Tan et al., 2012). Furthermore, in the colonial ascidian Botryllus schlosseri, telomerase activity is upregulated in early bud rudiments, and declines during zooid development (Laird and Weissman, 2004). Differences in the relative abundance of somatic versus stem cells might also determine variation in telomere length (Ojimi and Hidaka, 2010), but we cannot either discard recombination between homologous telomeres as a means to elongate telomeres (Liu et al., 2007). Even in plants, telomere restoration is only present in meristomatic and reproductive tissues, with exceptions in long-lived species (Flanary and Kletetschka, 2005; Watson and Riha, 2011 among other references), whereas in some algae it has been described telomerase activity during all life cycles, but large differences in telomerase activity have been found across algae groups (Fulnecková et al., 2013; Ševčíková et al., 2013). Further comparison of telomerase and stem cells in relation to regeneration in the analyzed populations of $C$. tenuispina would thus be of future interest.

The results presented here reveal the need for further research exploring the ecological and evolutionary significance of asexual reproduction and telomere elongation in clonal lineages. Future empirical studies measuring the success of Atlantic and Mediterranean populations should consider additional variables such as sexual reproductive success, as well as an evaluation of whether genetic diversity is fundamental to the maintenance of clonal populations, concepts that have been only theoretical explored for few authors as Weissman et al. (2009) and Marriage and Orive (2012). Those theoretical models for asexual species have evaluated and proposed that clones with large population sizes exhibit high successful levels (Weissman et al., 2009; Marriage and Orive, 2012). Nevertheless, further analysis regarding possible somatic deleterious mutations, as well as health and population size monitoring, is essential in order to understand the life expectancy of clonal populations.

\section{DATA ARCHIVING}

Data available from the Dryad Digital Repository: http://dx.doi.org/ $10.5061 /$ dryad. 305 c3.

\section{CONFLICT OF INTEREST}

The authors declare no conflict of interest.

\section{ACKNOWLEDGEMENTS}

We thank Dr Owen Wangensteen for his support during sampling and for his fruitful ideas. We are also indebted to Dr Jose Carlos Hernandez, Dr Sabrina Clemente and the staff of the University of La Laguna for their help and logistic support during sampling in Tenerife. We thank Susann Haraldsson, Umeå, for laboratory assistance with qPCR. This research was financially supported by a PhD fellowship FPI-MICINN (BES-2011-044154) (ACG), 
the European ASSEMBLY project (227799), the Swedish Royal Academy of Sciences (ACG) and the Spanish Government project CTM2010-22218-C02 and CTM2013-48163. The research was also supported by a 'Juan de la Cierva' contract from the Spanish Government (RPP) and by the Adlerbertska Research Foundation (HNS).

Abramoff MD, Magelhaes PJ, Ram SJ (2004). Image Processing with ImageJ. Biophotonics Int 11: 36-42.

Ally D, Ritland K, Otto SP (2008). Can clone size serve as a proxy for clone age? An exploration using microsatellite divergence in Populus tremuloides. Mol Ecol 17: 4897-4911.

Ally D, Ritland K, Otto SP (2010). Aging in a long-lived clonal tree. PLoS Biol 8: e1000454.

Alves LS, Pereira A, Ventura C (2002). Sexual and asexual reproduction of Coscinasterias tenuispina (Echinodermata: Asteroidea) from Rio de Janeiro, Brazil. Mar Biol 140. 95-101.

Arnaud-Haond S, Duarte CM, Diaz-Almela E, Marbà N, Sintes T, Serrão EA (2012). Implications of extreme life span in clonal organisms: millenary clones in meadows of the threatened seagrass Posidonia oceanica. PLoS One 7: e30454.

Barraclough TG, Fontaneto D, Ricci C, Herniou EA (2007). Evidence for inefficient selection against deleterious mutations in cytochrome oxidase I of asexual bdelloid rotifers. Mol Biol Evol 24: 1952-1962.

Barrett ELB, Burke TA, Hammers M, Komdeur J, Richardson DS (2013). Telomere length and dynamics predict mortality in a wild longitudinal study. Mol Ecol 22: 249-259.

Bize P, Criscuolo F, Metcalfe NB, Nasir L, Monaghan P (2009). Telomere dynamics rather than age predict life expectancy in the wild. Proc Biol Sci 276: 1679-1683.

Carney Almroth B, Sköld M, Sköld HN (2012). Gender differences in health and aging of Atlantic cod subject to size selective fishery. Biol Open 1: 922-928.

Cawthon RM (2002). Telomere measurement by quantitative PCR. Nucleic Acids Res $\mathbf{3 0}$ e47.

Crozier WJ (1921). Notes on some problems of adaptation. Biol Bull 41: 102-105.

Dahl MP, Pereyra RT, Lundälv T, André C (2012). Fine-scale spatial genetic structure and clonal distribution of the cold-water coral Lophelia pertusa. Coral Reefs 31 1135-1148.

Dixon P (2003). VEGAN, a package of R functions for community ecology. J Veg Sci 14 927-930.

Ebert TA, Russell MP, Gamba G, Bodnar A, Russel MP (2008). Growth, survival, and longevity estimates for the rock-boring sea urchin Echinometra lucunter lucunter (Echinodermata, Echinoidea) in Bermuda. Bull Mar Sci 82: 381-403.

Emson RH, Wilkie IC (1980). Fission and autonomy in echinoderms. Oceanography and Marine Biology: an Annual Review. Aberdeen University Press, 18: 155-250.

Epel ES, Blackburn EH, Lin J, Dhabhar FS, Adler NE, Morrow JD et al. (2004). Accelerated telomere shortening in response to life stress. Proc Natl Acad Sci USA 101: $17312-17315$.

Farzaneh-far R, Cawthon RM, Na B, Warren BS, Schiller NB, Whooley MA (2008). Prognostic value of leukocyte telomere length in patients with stable coronary artery disease: data from the Heart and Soul Study (R1). Arter Thromb Vasc Biol 28: 1379-1384.

Flanary BE, Kletetschka G (2005). Analysis of telomere length and telomerase activity in tree species of various life-spans, and with age in the bristlecone pine Pinus longaeva. Biogerontology 6: 101-111.

Fulnecková J, Sevcíková T, Fajkus J, Lukesová A, Lukes M, Vlcek C et al. (2013). A broad phylogenetic survey unveils the diversity and evolution of telomeres in eukaryotes. Genome Biol Evol 5: 468-483.

Garcia-Cisneros A, Valero-Jiménez C, Palacín C, Pérez-Portela R (2013). Characterization of thirty two microsatellite loci for three Atlanto-Mediterranean echinoderm species. Conserv Genet Resour 5: 749-753.

Gerlach G, Jueterbock A, Kraemer P, Deppermann J, Harmand P (2010). Calculations of population differentiation based on GST and D: forget GST but not all of statistics!. Mol Ecol 19: 3845-3852.

Gomes NMV, Shay JW, Wright WE (2010). Telomere biology in Metazoa. FEBS Lett 584: $3741-3751$

Goudet J (2005). Hierfstat, a package for $r$ to compute and test hierarchical F-statistics. Mol Ecol Notes 5: 184-186.

Goudie F, Allsopp MH, Beekman M, Oxley PR, Lim J, Oldroyd BP (2012). Maintenance and loss of heterozygosity in a thelytokous lineage of honey bees (Apis mellifera capensis). Evolution 66: 1897-1906.

Grabowski P, Hultdin M, Karlsson K, Tobin G, Aleskog A, Thunberg U et al. (2005). Telomere length as a prognostic parameter in chronic lymphocytic leukemia with special reference to VH gene mutation status. Blood 105: 4807-4812.
Haramoto S, Komatsu M, Yamazaki Y (2007). Patterns of asexual reproduction in the fissiparous seastar Coscinasterias acutispina (Asteroidea: Echinodermata) in Japan. Zool Sci 24: 1075-1081.

Herbig U, Ferreira M, Condel L, Carey D, Sedivy JM (2006). Cellular senescence in aging primates. Science 311: 1257.

Hernroth B, Farahani F, Brunborg G, Dupont S, Dejmek A, Sköld HN (2010). Possibility of mixed progenitor cells in sea star arm regeneration. J Exp Zool 314: 457-468.

Hoffman JI, Forcada J, Trathan PN, Amos W (2007). Female fur seals show active choice for males that are heterozygous and unrelated. Nature 445: 912-914.

Horn T, Robertson BC, Gemmell NJ (2010). The use of telomere length in ecology and evolutionary biology. Heredity 105: 497-506.

Hörandl E (2009). A combinational theory for maintenance of sex. Heredity 103 . $445-457$.

Jost L (2008). G(ST) and its relatives do not measure differentiation. Mol Ecol 17: 4015-4026.

Karako S, Achituv Y, Perl-Treves R, Katcoff D (2002). Asterina burtoni (Asteroidea; Echinodermata) in the Mediterranean and the Red Sea: does asexual reproduction enhance colonization? Mar Ecol Prog Ser 234: 139-145.

Klapper W, Kühne K, Singh KK, Heidorn K, Parwaresch R, Krupp G (1998). Longevity of lobsters is linked to ubiquitous telomerase expression. FEBS Lett 439: 143-146.

Kotrschal A, IImonen P, Penn DJ (2007). Stress impacts telomere dynamics. Biol Lett $\mathbf{3}$ : 128-130.

Laird DJ, Weissman IL (2004). Telomerase maintained in self-renewing tissues during serial regeneration of the urochordate Botryllus schlosseri. Dev Biol 273: 185-194.

Lawrence J (2013). Starfish: Biology and Ecology of the Asteroidea. Johns Hopkins University Press: Baltimore.

Liu L, Bailey SM, Okuka M, Muñoz P, Li C, Zhou L et al. (2007). Telomere lengthening early in development. Nat Cell Biol 9: 1436-1441.

Marriage TN, Orive ME (2012). Mutation-selection balance and mixed mating with asexual reproduction. J Theor Biol 308: 25-35.

Meirmans PG, Van Tienderen PH (2004). Genotype and genodive: two programs for the analysis of genetic diversity of asexual organisms. Mol Ecol Notes 4: 792-794.

Ojimi MC, Hidaka M (2010). Comparison of telomere length among different life cycle stages of the jellyfish Cassiopea andromeda. Mar Biol 157: 2279-2287.

O'Callaghan N, Dhillon V, Thomas P, Fenech M (2008). A quantitative real-time PCR method for absolute telomere length. Biotechniques 44: 807-809.

Paland S, Lynch M (2006). Transitions to asexuality result in excess amino acid substitutions. Science 311: 990-992.

Reusch TBH, Boström C, Stam WT, Olsen JL (1999). An ancient eelgrass clone in the Baltic. Mar Ecol Prog Ser 183: 301-304.

Schaetzlein S, Lucas-Hahn A, Lemme E, Kues WA, Dorsch M, Manns MP et al. (2004). Telomere length is reset during early mammalian embryogenesis. Proc Natl Acad Sci USA 101: 8034-8038.

Schwander T, Crespi BJ (2009). Twigs on the tree of life? Neutral and selective models for integrating macroevolutionary patterns with microevolutionary processes in the analysis of asexuality. $\mathrm{Mol} \mathrm{Ecol} 18: 28-42$.

Sköld HN, Asplund ME, Wood C a, Bishop JDD (2011). Telomerase deficiency in a colonial ascidian after prolonged asexual propagation. J Exp Zool B Mol Dev Evol 316: 276-283.

Sköld HN, Obst M (2011). Potential for clonal animals in longevity and ageing studies. Biogerontology 12: 387-396.

Stenberg P, Lundmark M, Saura A (2003). mlgsim: a program for detecting clones using a simulation approach. Mol Ecol Notes 3: 329-331.

Ševčíková T, Bišová K, Fojtová M, Lukešová A, Hrčková K, Sýkorová E (2013). Completion of cell division is associated with maximum telomerase activity in naturally synchronized cultures of the green alga Desmodesmus quadricauda. FEBS Lett 587: 743-748.

Tan TCJ, Rahman R, Jaber-Hijazi F, Felix DA, Chen C, Louis EJ et al. (2012). Telomere maintenance and telomerase activity are differentially regulated in asexual and sexual worms. Proc Natl Acad Sci U S A 109: 4209-4214.

Ventura CRR, Alves SLS, Maurício CEP, Silva EP (2004). Reproduction and population genetics of Coscinasterias tenuispina. (Asteroidea: Asteriidae ) on the Brazilian coast. In: Nebelsick JH, Heinzeller T (eds), Echinoderms: Munchen Proceedings of the 11th International Echinoderm Conference, 6-10 October 2003. Taylor \& Francis: Munich, Germany2004: Munich. pp 73-77.

Von Zglinicki T (2002). Oxidative stress shortens telomeres. Trends Biochem Sci 27: 339-344.

Watson JM, Riha K (2011). Telomeres, aging, and plants: from weeds to Methuselah - a mini-review. Gerontology 57: 129-136.

Weissman DB, Desai MM, Fisher DS, Feldman MW (2009). The rate at which asexual populations cross fitness valleys. Theor Popul Biol 75: 286-300.

Supplementary Information accompanies this paper on Heredity website (http://www.nature.com/hdy) 